\title{
Association of Maternal Demographic Characteristics With Breastfeeding of Low Birth Weight Infants in Ohio in 2012
}

Raymond I. Okeke Jr., MPH ${ }^{1}$

Share this:

Sara J. Paton, $\mathrm{PhD}^{2}$

Linda J. Smith, MPH, IBCLC, RLC ${ }^{3}$

Naila Khalil, MBBS, MPH, $\mathrm{PhD}^{4}$

Background: Breastfeeding is the biologic norm for infant feeding and vitally important for newborns especially the low birth weight (LBW) infant population who have higher morbidity and mortality. For LBW infants in Ohio, prevalence of breastfeeding with maternal sociodemographic factors is not fully known.

Methods: The 2012 Ohio Vital Statistics Birth data including 10,571 LBW infants compiled by the Ohio Department of Health were analyzed (8.5\% of the total Ohio births in 2012). Descriptive sociodemographic characteristics were summarized by breastfeeding status. Association of breastfeeding in LBW infants was computed using univariate and multivariate logistic regression (LR) analyses.

Results: Among the LBW infants, 60\% were breastfed. Breastfeeding was significantly higher in LBW infants with increasing maternal age and mothers with more prenatal visits. Within all three race categories (White, African American, others), more mothers breastfed LBW babies. However, among LBW babies being breastfed, the highest proportion was in White families. Mother's education greater than high school, being married, not on Medicaid, and being nonsmoker were associated with higher rates of breastfeeding. In multivariate LR, increased prenatal visits $(\mathrm{OR}=1.03,95 \% \mathrm{CI}[1.02,1.04], \mathrm{p}<.001)$ were associated with a higher breastfeeding percentage compared to mothers of African American race, LBW babies of "other race" had 71\% significantly higher breastfeeding percentage $(\mathrm{OR}=1.71,95 \% \mathrm{CI}[1.39,2.11]$, $p<.001)$. Having a high school or higher education (OR $=1.52,95 \%$ CI [1.37, 1.69], $p<$ $.001)$, married $(\mathrm{OR}=1.69,95 \% \mathrm{CI}[1.52,1.87], \mathrm{p}<.001)$, not on Medicaid $(\mathrm{OR}=1.59$, $95 \%$ CI $[1.44,1.75], p<.001)$, and not smoking $(\mathrm{OR}=2.04,95 \% \mathrm{CI}[1.84,2.26], \mathrm{p}<$ .001) were associated with higher breastfeeding percentage.

Conclusion: The prevalence of breastfeeding initiation in LBW infants is lower than normalweight infants. Mothers' sociodemographic characteristics affect breastfeeding in LBW infants in the same way they affect mothers with normal-weight infants. Targeted efforts during antenatal care to promote breastfeeding would help LBW infants.

Keywords: breastfeeding; lactation; low birth weight

According to the World Health Organization (WHO), low birth weight (LBW) is birth weight less than 2,500 g (United Nations Children's Fund \& WHO, 2004). Preterm birth and intrauterine growth retardation are two causes of LBW (Kelley \& Podja, 2000; Khan, Arbab, Murad, Khan, \& Abdullah, 2014; Nohr et al., 2008). Risk factors for LBW include mother's low

\footnotetext{
1. okekeri@slu.edu

2. sara.paton@wright.edu

3. Lindaj@bflrc.com

4. naila.khalil@wright.edu
}

education, mother's younger or older age, race, poverty, unmarried or early marriage, and smoking before and during pregnancy (Khan et al., 2014).

Breastfeeding is the optimal way of feeding a baby. Lactation is a metabolically intensive process requiring the breakdown of maternal nutrients which transfer to the fetus (Stuebe \& Rich-Edwards, 2009). Mother's age, race, education and marital status, household composition, living arrangements, employment, and maternity care, and socioeconomic status affect breastfeeding intention (Lewallen \& Street, 2010; Lind, Perrine, Li, Scanlon, \& Grummer-Strawn, 2014; 
the Special Supplemental Nutrition Program for Women, Infants, and Children).

This study evaluates association between sociodemographic factors affecting mothers of LBW infants and prevalence of breastfeeding in LBW infants in Ohio.

\section{Method}

The 2012 Ohio Vital Statistics Birth data including 10,571 LBW infants compiled by the Ohio Department of Health were analyzed $(8.5 \%$ of the total Ohio births in 2012). Sociodemographic characteristics were summarized by breastfeeding status, defined as mothers who initiated breastfeeding before baby leaves the hospital. Association of breastfeeding in LBW infants was computed using univariate and multivariate logistic regression (LR) analyses. Descriptive analysis included comparison of covariates across the two categories of breastfeeding (yes/no). The significance level for all hypothesis tests was $\alpha=.05$ (two-tailed).

After checking normality for all continuous variables, measures of centrality (mean) and dispersion (standard deviation) were computed and tested using Student's $t$ test. For categorical variables, frequency distributions were calculated and tested across breastfeeding categories using Pearson chi-square analysis. Univariate LR was completed to assess association of each predictor variable with breastfeeding (yes/no; no $=$ referent). The result was computed as odds ratio of breastfeeding, with $95 \%$ confidence interval and $p$ values. To assess independent association of individual predictor variables with breastfeeding after controlling for covariates, multivariate (LR) analysis was completed. In multivariate LR, mother's age, number of prenatal visits, mother's race, education, marital status, insurance type, and smoking status during third trimester were analyzed.

\section{Results}

The total number of LBW infants was 10,$571 ; 60 \%$ of these initiated breastfeeding. Mothers who breastfed were on average older than mothers who did not breastfeed (28 years vs. 26 years, respectively; $p<.001$; Table 1). In univariate LR, increasing age of mothers was associated with $4 \%$ higher prevalence of breastfeeding (odds ratio $[O R]=1.04,95 \%$ confidence interval $[\mathrm{CI}]$ $[1.03,1.05], p<.001)$, but this association was not significant in multivariate LR. Among LBW infants, the mean number of prenatal visits of the mothers who breastfed was higher than for mothers who did not breastfeed (10 visits vs. 9 visits, respectively; $p<.001$ ).
Higher number of prenatal visits was associated with $4 \%$ higher prevalence of breastfeeding $(O R=1.04,95 \%$ CI $[1.03,1.05], p<.001)$ in bivariate LR and $3 \%$ higher breastfeeding prevalence $(\mathrm{OR}=1.03,95 \% \mathrm{CI}[1.02$, 1.03], $p<.001)$ in multivariate LR.

Among mothers who breastfed, more White mothers breastfed (68\%) than African American mothers (25\%) and mothers of other races (7\%; chi-square $p<.001)$. In univariate regression, breastfeeding was 33\% higher in Whites $(\mathrm{OR}=1.33,95 \% \mathrm{CI}[1.22,1.45], p<.001)$ and $151 \%$ higher in "others" $(\mathrm{OR}=2.51,95 \% \mathrm{CI}$ [2.06, 3.06], $p<.001$ ). However, in multivariate (LR), compared to African Americans, only "other" LBW infants showed a significantly higher breastfeeding prevalence $(\mathrm{OR}=1.51,95 \% \mathrm{CI}[1.25,1.81], p<.001)$.

In LBW babies, mothers having at least a high school education breastfeed more $(85 \%$ vs. less educated mothers [15\%], respectively; $p<.001$ ). In univariate and multivariate LR, high school or greater education was associated with a $124 \%$ higher breastfeeding prevalence $(\mathrm{OR}=2.24,95 \% \mathrm{CI}[2.03,2.46], p<.001)$ and $52 \%$ higher breastfeeding prevalence $(\mathrm{OR}=1.52,95 \% \mathrm{CI}$ $[1.37,1.69], p<.001)$, respectively. A higher proportion of mothers who breastfed were married ( $54 \%$ vs. $46 \%$, respectively; $p<.001)$. Being married was associated with a $163 \%$ higher breastfeeding prevalence $(O R=2.43$, $95 \%$ CI $[2.25,2.86], p<.001)$ in bivariate LR and $169 \%$ higher breastfeeding prevalence $(\mathrm{OR}=1.69,95 \% \mathrm{CI}$ $[1.52,1.87], p<.001)$ in multivariate LR.

Among mothers who breastfed, a higher proportion was using insurance other than Medicaid, (40\% on Medicaid vs. $60 \%$ on other insurance; $p<.001)$. Having another insurance than Medicaid was associated with a $156 \%$ higher prevalence of breastfeeding $(\mathrm{OR}=2.56,95 \% \mathrm{CI}$ $[2.37,2.78], p<.001)$ in univariate LR and $59 \%$ higher breastfeeding prevalence $(O R=1.59,95 \%$ CI [1.44, 1.75], $p<.001)$ in multivariate LR. Among LBW infants born in Ohio in 2012, more mothers who breastfed were nonsmokers ( $85 \%$ vs. smokers $15 \%$; $p<.001)$. As compared to smokers, nonsmoking mothers had 191\% higher prevalence of breastfeeding $(\mathrm{OR}=2.91,95 \% \mathrm{CI}$ $[2.65,3.19], p<.001)$ in univariate LR and $104 \%$ higher breastfeeding prevalence $(\mathrm{OR}=2.04,95 \%$ CI $[1.84$, 2.26], $p<.001)$ in multivariate LR.

\section{Discussion}

Maternal sociodemographic factors that were positively associated with breastfeeding of LBW infants in Ohio included mothers completing more prenatal visits, being married, education to at least high school, belonging to 
Table 1. Distribution of Sociodemographic Characteristics and Their Association With Breastfeeding in Low Birth Weight (LBW) Infants in Ohio (2012)

\begin{tabular}{|c|c|c|c|c|c|c|c|c|}
\hline \multirow[b]{2}{*}{ Characteristics } & \multicolumn{4}{|c|}{ Descriptive Characteristics } & \multicolumn{2}{|c|}{ Univariate $O R$} & \multicolumn{2}{|c|}{ Multivariate $O R$} \\
\hline & $\begin{array}{c}\text { Total } \\
(\mathrm{N}=10,571) \\
\end{array}$ & $\begin{array}{l}\text { No Breastfeeding } \\
(n=4,174 ; 40 \%)\end{array}$ & $\begin{array}{l}\text { Yes Breastfeeding } \\
(n=6,394 ; 60 \%) \\
\end{array}$ & $p$ Value & OR $(95 \% \mathrm{CI})$ & $p$ Value & OR $(95 \% \mathrm{CI})$ & $p$ Value \\
\hline Mother's age (years), M (SD) & $27(6)$ & $26(6)$ & $28(6)$ & $<.001$ & $1.04[1.03,1.05]$ & $<.001$ & $0.99[0.98,1.00]$ & .473 \\
\hline $\begin{array}{l}\text { Number of prenatal visits, } \\
\text { M (SD) }\end{array}$ & $8(6)$ & $8(5)$ & $9(6)$ & $<.001$ & $1.04[1.03,1.05]$ & $<.001$ & $1.03[1.02,1.03]$ & $<.001$ \\
\hline \multicolumn{9}{|l|}{ Mother's race, $n(\%)$} \\
\hline African American & 2,895 (27) & $1,310\left(31^{\mathrm{a}} ; 45\right)$ & $1,585(25 ; 55)$ & & Reference & & Reference & \\
\hline White & $7,067(67)$ & $2,714(65 ; 38)$ & $4,353(68 ; 62)$ & & $1.33[1.22,1.45]$ & $<.001$ & $0.98[0.89,1.08]$ & .700 \\
\hline Other $^{\mathrm{b}}$ & $606(6)$ & $150(4 ; 40)$ & $456(7 ; 60)$ & $<.001$ & $2.51[2.06,3.06]$ & $<.001$ & $1.71[1.39,2.11]$ & $<.001$ \\
\hline \multicolumn{9}{|l|}{ Mother's education, $n$ (\%) } \\
\hline Less than high school & $2,119(20)$ & $1,170(28 ; 55)$ & $949(15 ; 45)$ & $<.001$ & Reference & & Reference & \\
\hline $\begin{array}{l}\text { High school graduate } \\
\text { or higher }\end{array}$ & $8,449(80)$ & $3,004(72 ; 36)$ & $5,445(85 ; 64)$ & & $2.24[2.03,2.46]$ & $<.001$ & $1.52[1.37,1.69]$ & $<.001$ \\
\hline \multicolumn{9}{|l|}{ Mother's marital status, $n(\%)$} \\
\hline Not married & $5,792(55)$ & $2,874(69 ; 50)$ & $2,918(46 ; 50)$ & $<.001$ & Reference & & Reference & \\
\hline Married & $4,776(45)$ & $1,300(31 ; 27)$ & $3,476(54 ; 73)$ & & $2.63[2.43,2.86]$ & $<.001$ & $1.69[1.52,1.87]$ & $<.001$ \\
\hline \multicolumn{9}{|l|}{ Mother's insurance, $n(\%)$} \\
\hline Medicaid & $5,213(49)$ & $2642(63 ; 51)$ & $2571(40 ; 49)$ & $<.001$ & Reference & $<.001$ & Reference & \\
\hline Other insurance & $5,355(51)$ & $1532(37 ; 29)$ & $3823(60 ; 71)$ & & $2.56[2.37,2.78]$ & & $1.59[1.44,1.75]$ & $<.001$ \\
\hline \multicolumn{9}{|l|}{ Smoking third trimester, $n(\%)$} \\
\hline Smoker & $2,443(23)$ & $1,452(35 ; 59)$ & $991(15 ;(41)$ & & Reference & & Reference & \\
\hline Nonsmoker & $8,125(77)$ & $2,722(65 ; 33)$ & $5,403(85 ; 67)$ & $<.001$ & $2.91[2.65,3.19]$ & $<.001$ & $2.04[1.84,2.26]$ & $<.001$ \\
\hline
\end{tabular}

Note. $\mathrm{OR}=$ odds ratio; $\mathrm{CI}=$ confidence interval.

${ }^{a}$ Where two percentages are provided, the first one is column percentage and second is row percentage (col \%; row \%)

${ }^{b}$ Other races compose of the following: Native American (American Indian, includes Alaskan, Aleut, Eskimo), Chinese, Japanese, Hawaiian (includes part Hawaiian), Filipino, Other Asian or Pacific Islander, Other non-White (father's race only, mothers imputed to 1-8), not classifiable (father's race only, mothers imputed to 1-8). 
"other" race, being not on Medicaid, and a nonsmoker. These findings affirm those in Spencer and Grassley (2013) as well as in Forste, Weiss, and Lippincott (2001) who stressed that sociodemographic factors influence breastfeeding practices.

This analysis was restricted to LBW infants. Hill, Ledbetter, and Kavanaugh (1997) reported that LBW mothers face more challenges in breastfeeding than mothers of non-LBW infants because of problems with latching on and associated feeding-related morbidities in the infants. The current analysis supports this: Only $60 \%$ of LBW infants initiated breastfeeding in Ohio in 2012. In 2012, 71.9\% of all infants in Ohio were ever breastfed compared to a national rate of $80.1 \%$ (Centers for Disease Control and Prevention [CDC], 2016).

LBW infants are breastfed less than non-LBW infants. It was noted, at least in univariate analysis in this study, that as mothers' age, the prevalence of breastfeeding increases. Established factors associated with increasing breastfeeding with mother's age include increased education and marriage (Spencer \& Grassley, 2013). Mothers with a higher number of prenatal visits breastfeed more. This finding is consistent with literature which theorizes that access to prenatal care improves breastfeeding intention, initiation, duration, and exclusivity (Agrasada, Ewald, Kylberg, \& Gustafsson, 2011).

Among mothers who breastfed, predominant proportion were White mothers (68\%). In contrast, only $25 \%$ of African American mothers and 7\% of mothers of other races breastfed LBW infants. Literature suggests that the low prevalence of breastfeeding in African American population is because of cultural reluctance to breastfeed (Lewallen \& Street, 2010). The findings of this study are consistent with that hypothesis even among LBW infants.

According to Forste et al. (2001), there are restrictions to the types of jobs and health services mothers can access as well as the neighborhoods they live in because of their educational status, which can affect their breastfeeding patterns. Findings in this study are consistent with this concept because more mothers with high school diplomas or higher education breastfed than mothers with less than high school education.

In this study, married women had a higher prevalence of breastfeeding compared to women who were unmarried. Spousal support plays a key role in encouraging intention to breastfeed and the initiation of breastfeeding. Studies have shown that the type of family unit determines exclusivity and duration of breastfeeding after initiation (CDC, 2013). According to this study, among the mothers who breastfed, a higher proportion had other insurance than Medicaid. Woo et al. (2012) suggest that the prevalence of breastfeeding is lower in a population who are of a lower socioeconomic status, have less favorable employment opportunities, lack support, and are beneficiaries of supplementary feeding. The findings of the current analysis are consistent with this outcome in LBW infants. In this analysis, mothers who were nonsmokers had a higher prevalence of breastfeeding initiation than smokers. As indicated in other studies, mothers who smoke tend to breastfeed less than nonsmoking mothers (Al-Sahab, Lanes, Feldman, \& Tamim, 2010).

Our results should be interpreted with caution. The sample size was restricted to LBW infants: The data for breastfeeding only cover initiation and does not include data on duration or exclusivity of breastfeeding. Although the study's findings are applicable to LBW infants in Ohio, it is not assumed that they can be generalized to other states.

In conclusion, maternal sociodemographic characteristics may play a role in breastfeeding among mothers of LBW infants. Results of this study suggest that mothers of LBW infants breastfeed at a lower rate than mothers of normalweight infants. Given that breastfeeding is critical for these infants, the public health community should help promote breastfeeding within this population. This study also shows that factors that support breastfeeding LBW infants are similar as those for normal-weight infants.

\section{References}

Agrasada, G. V., Ewald, U., Kylberg, E., \& Gustafsson, J. (2011). Exclusive breastfeeding of low birth weight infants for the first six months: Infant morbidity and maternal and infant anthropometry. Asia Pacific Journal of Clinical Nutrition, 20(1), 62-68.

Al-Sahab, B., Lanes, A., Feldman, M., \& Tamim, H. (2010). Prevalence and predictors of 6-month exclusive breastfeeding among Canadian women: A national survey. BMC Pediatrics, 10(1), 20.

Centers for Disease Control and Prevention. (2013). Progress in increasing breastfeeding and reducing racial/ethnic differencesUnited States, 2000-2008 births. Morbidity and Mortality Weekly Report, 62(5), 77-80.

Centers for Disease Control and Prevention. (2016). Rates of any and exclusive breastfeeding by state among children born in 2012 (Percentage +/- half 95\% confidence interval) [Data file]. Retrieved from https://www.cdc.gov/breastfeeding/data/ nis_data/rates-any-exclusive-bf-state-2012.htm

Forste, R., Weiss, J., \& Lippincott, E. (2001). The decision to breastfeed in the United States: Does race matter? Pediatrics, 108(2), 291-296.

Hill, P. D., Ledbetter, R. J., \& Kavanaugh, K. L. (1997). Breastfeeding patterns of low-birth-weight infants after hospital discharge. Journal of Obstetric, Gynecologic, and Neonatal Nursing, 26(2), 189-197.

Kelley, L., \& Pojda, J. (2000). Low birth weight (Nutrition Policy Paper No. 18). Geneva, Switzerland: United Nations. 
Khan, M. W., Arbab, M., Murad, M., Khan, M. B., \& Abdullah, S. (2014). Study of factors affecting and causing low birth weight. Journal of Scientific Research, 6(2), 387-394.

Lewallen, L. P., \& Street, D. J. (2010). Initiating and sustaining breastfeeding in African American women. Journal of Obstetric, Gynecologic, and Neonatal Nursing, 39(6), 667-674.

Lind, J. N., Perrine, C. G., Li, R., Scanlon, K. S., \& GrummerStrawn, L. M. (2014). Racial disparities in access to maternity care practices that support breastfeeding-United States, 2011. MMWR, 63(33), 725-728.

Nohr, E. A., Vaeth, M., Baker, J. L., Sørensen, T. I. A., Olsen, J., \& Rasmussen, K. M. (2008). Combined associations of pregnancy body mass index and gestational weight gain with the outcome of pregnancy. The American Journal of Clinical Nutrition, 87, 1750-1759.
Spencer, B. S., \& Grassley, J. S. (2013). African American women and breastfeeding: An integrative literature review. Health Care for Women International, 34, 607-625.

Stuebe, A. M., \& Rich-Edwards, J. W. (2009). The reset hypothesis: Lactation and maternal metabolism. American Journal of Perinatology, 26, 81-88.

United Nations Children's Fund \& World Health Organization. (2004). Low birth weight, country, regional and global estimates. New York, NY: United Nations Children's Fund.

Woo, J. G., Guerrero, M. L., Ruiz-Palacios, G. M., Peng, Y., Herbers, P. M., Yao, W., . . Morrow, A. L. (2012). Specific infant feeding practices do not consistently explain variation in anthropometry at age 1 year in urban United States, Mexico, and China cohorts. The Journal of Nutrition, 143, 166-174.

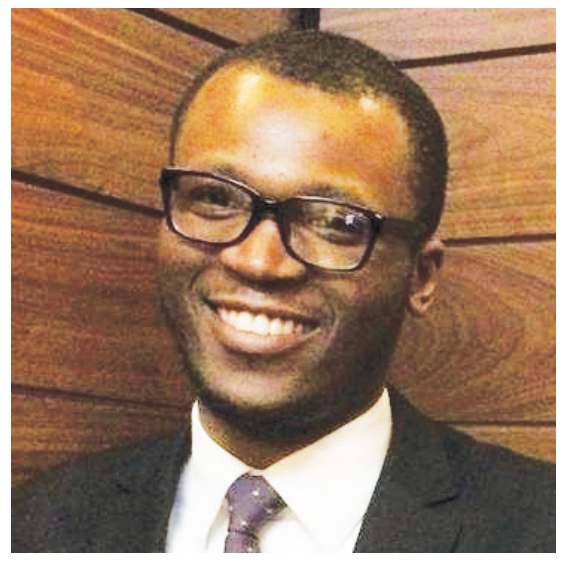

Raymond Okeke Jr., MPH, is a medical student at Saint Louis University School of Medicine, Class of 2019. He completed his MPH at Wright State University (WSU) and is fluent in three languages.

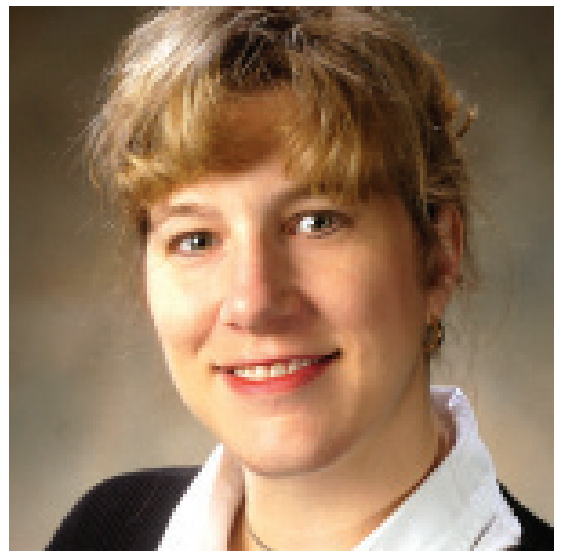

Sara J. Paton, $\mathrm{PhD}$, is an associate professor of epidemiology in the Center for Global Health and has a joint appointment with the WSU Department of Community Health and Public Health-Dayton \& Montgomery County. 

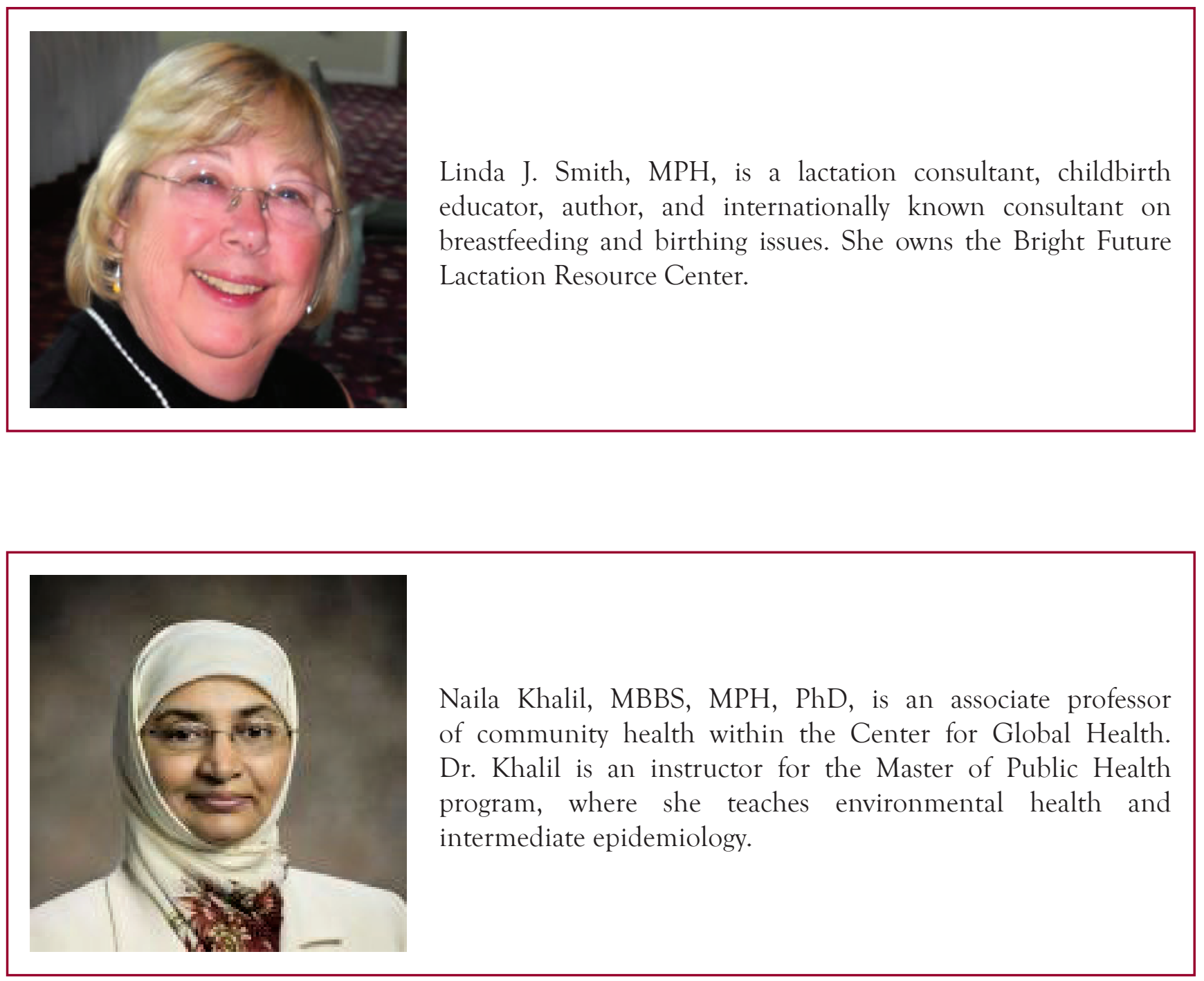

\section{Sex Discrimination Regulations From the Department of Labor}

The U.S. Department of Labor Office of Federal Contract Compliance Programs has published updated sex discrimination regulations for federal contractors. The final rule updates OFCCP's sex discrimination regulations to make them consistent with current law and makes explicit the protections against compensation discrimination; sexually hostile work environments; discrimination based on pregnancy, childbirth or related medical conditions; and discrimination based on unlawful sex stereotypes, gender identity, and transgender status. The updated rule lists lactation as a pregnancy-related medical condition. https://www.dol.gov/ofccp/SexDiscrimination.html

Source: USBC 